\title{
ZONE OF MINIMAL CONFLICT RECORDED USING MODIFIED TECHNIQUE: A CASE REPORT
}

\author{
Apurva Sharma ${ }^{1}$, Manjit Kumar ${ }^{2}$, Amrit Khosla ${ }^{3}$, Shailesh Jain ${ }^{4}$, Navjot Kaur ${ }^{5}$, Ashu Jhamb ${ }^{6}$ \\ ${ }^{1}$ Post Graduate Student, Department of Prosthodontics, Bhojia Dental College and Hospital, Himachal Pradesh, India \\ ${ }^{2}$ Prof. \& Head, Department of Prosthodontics, Bhojia Dental College and Hospital, Himachal Pradesh, India \\ ${ }^{3}$ Professor, Department of Prosthodontics, Bhojia Dental College and Hospital, Himachal Pradesh, India \\ ${ }^{4}$ Reader, Department of Prosthodontics, Bhojia Dental College and Hospital, Himachal Pradesh, India \\ ${ }^{5}$ Post Graduate Student, Department Of Prosthodontics, Bhojia Dental College and Hospital, Himachal Pradesh, India \\ ${ }^{6}$ Reader, Department of Conservative dentistry \& Endodontics, Bhojia Dental College and Hospital, Himachal Pradesh, India
}

\begin{abstract}
\begin{tabular}{l|l}
\hline &
\end{tabular}
ABSTRACT

When all the remaining natural teeth are removed, there exists a space within the oral cavity that can be called the potential denture space. Within this denture space there is an area that has been termed the neutral zone. Neutral zone is defined as that area or position within the oral cavity where the forces between the tongue and cheeks or lips are equal. Thus making it the zone of minimal conflict, where the function of musculature will not unseat the denture and help in stabilizing the denture.In the present case the neutral zone was recorded using admix technique and impression compound as the material of choice.
\end{abstract}

Key Words: Denture Space, Neutral zone,mandible atrophies, admix material, admixed technique.

\section{INTRODUCTION}

Complete dentures are mechanical devices meant to function in oral cavity which is a dynamic environment, owing to the highly evolved and complex neuromusculature surrounding it. The harmonious coexistence of the complete denture prosthesis with the neuromuscular functioning is the foundation of a successful denture.

Fabrication of mandibular denture is usually more challenging with the patient reporting with complaints of pain and looseness. The rate of atrophy in mandible is greater than that of the maxilla rendering residual ridge for retention and support.

To achieve the goal of providing a stable and retentive complete denture to patients with mandible atrophy, the positioning of artificial teeth assures a very important role. After all of the natural teeth have been lost a void exists in the oral cavity which is the "potential denture space".
It is imperative and advantageous to position the artificial teeth in this space, so that the forces exerted by the musculature serve to stabilize the denture rather than unseat it.

The neutral zone has been defined as the area in the mouth where during function, the forces of the tongue pressing outwards are neutralized by the forces of the cheeks and lips pressing inwards. It is that area or position where the forces between the tongue and cheeks or lips are equal. ${ }^{1}$

Various materials like Tissue conditioners $^{2}$, Impression compound ${ }^{3}$, Waxes $^{4}$ and Impression plaster ${ }^{5}$ have been advocated to record neutral zone which has their own advantages as well as disadvantages.In the present case report the neutral zone was located and recorded using admix technique with impression compound as the material of choice.

\section{CASE REPORT}

Maxillary and mandibular primary impressions were made in stock 
tray using thermoplastic impression materialimpression compound (CE ROLEX, Delhi, India). Custom trays were fabricated in autopolymerisingresin(DPI-RR Cold cure, Mumbai, India) and secondary impression was recorded with the help of admixed technique and irreversible hydrocolloid material(Prime Chrome, Thane, India) (Fig.1 ). For admixed technique, medium fusing impression
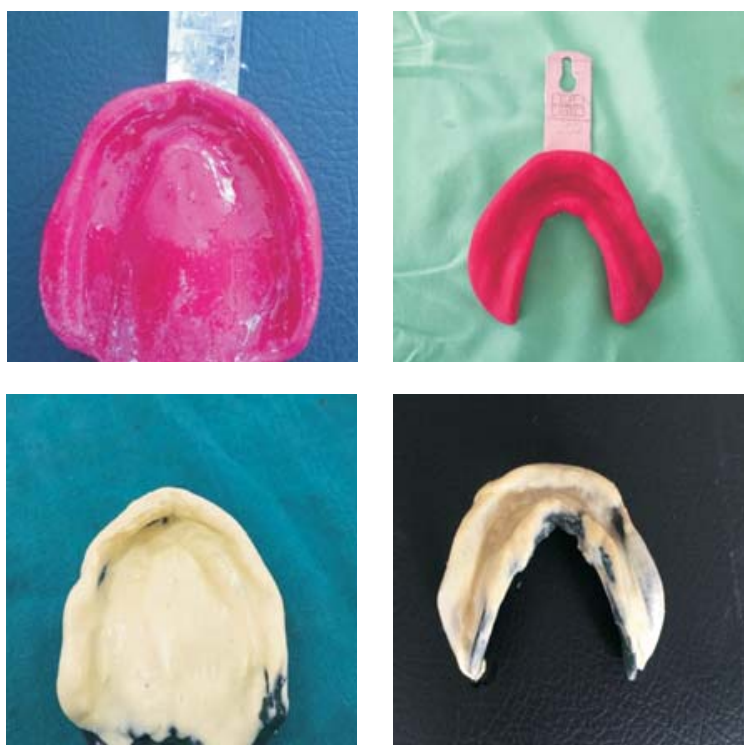

Figure 1 - Impression making

compound and low fusing impression compound-green stick (DPI Pinnacle, Mumbai, India) were mixed together in ratio of 3:7 by weight and placed in a bowl of water at $60^{\circ} \mathrm{C}$ and kneaded to a homogenous mass that provides a working time of about 90 seconds. ${ }^{6}$ Jaw relation were then recorded using conventional occlusal rims made of modeling wax and occlusal blocks were mounted on non-adjustable articulator.

An additional autopolymerizing resin mandibular denture base was fabricated and was attached with retentive loops made of thin orthodontic wire in the centre (Fig. 2). Two vertical pillars made of an additional auto polymerizingresin were placed in the first molar region at established vertical dimension. Retentive loops extended posterior to vertical pillars. This denture base was placed in the mouth,
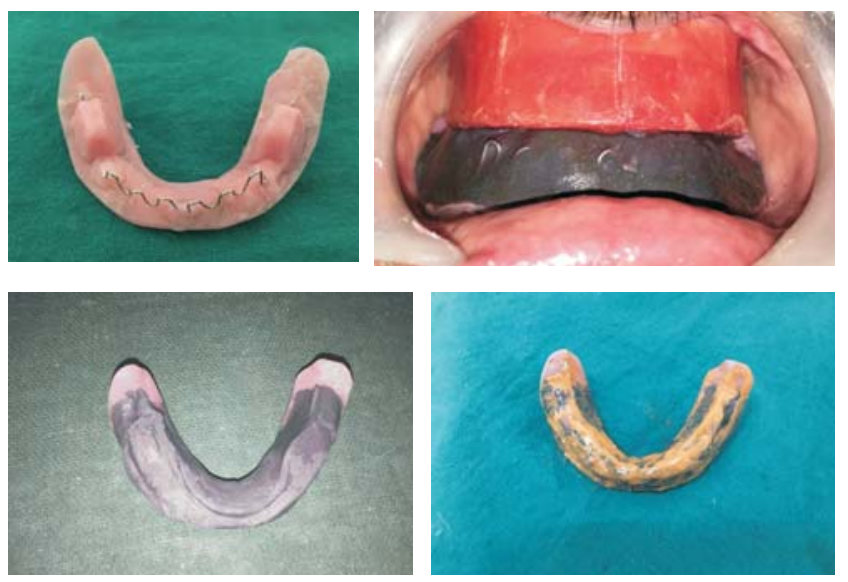

Figure 2 - Steps of modified technique for recording neutral zone

checked for stability and ensured that loops and vertical pillars did not interfere with muscle movements during function. ${ }^{5}$ Maxillary occlusal rim was placed back in the patient's mouth.

Impression compound and green stick material (DPI Pinnacle, Mumbai, India)were mixed together in ratio of 3:7. This material was placed over the denture base and inserted in the mouth (Fig.2). Patient was instructed to perform all muscle functions by sucking and swallowing movements and by producing exaggerated 'EEE' and 'OOO' sounds. ${ }^{5}$ Excess material if any will be displaced upward in the upper denture space from where it could be easily removed.

In case of insufficient material, additions could easily be made using extra material and the process was repeated. $0.5 \mathrm{~mm}$ of scraping was done on the buccal and lingual side of the impression and finer details of the contours recorded using Zinc Oxide-Eugenol impression material (Fig.2).

The impression of denture space was placed over the mandibular master cast. Indexing was done on lateral sides and tongue region in mandibularcast. Lingual indices of this denture space was made using silicone putty (Zetaplus Putty C-silicone,Italy). Putty was adapted into the tongue space of the neutral zone record so that it was in level of occlusal plane of record and extends over the posterior land area of cast. Likewise facial index was developed along the facial contours of the neutral zone record. Once polymerized, 
putty indices were removed from the cast. Previous denture base with wax rim was placed on the articulator. Occlusal (wax) rim was removed from the denture base and putty index was adapted in place over this denture base. Then, these putty indices were filled with wax (Fig.3).
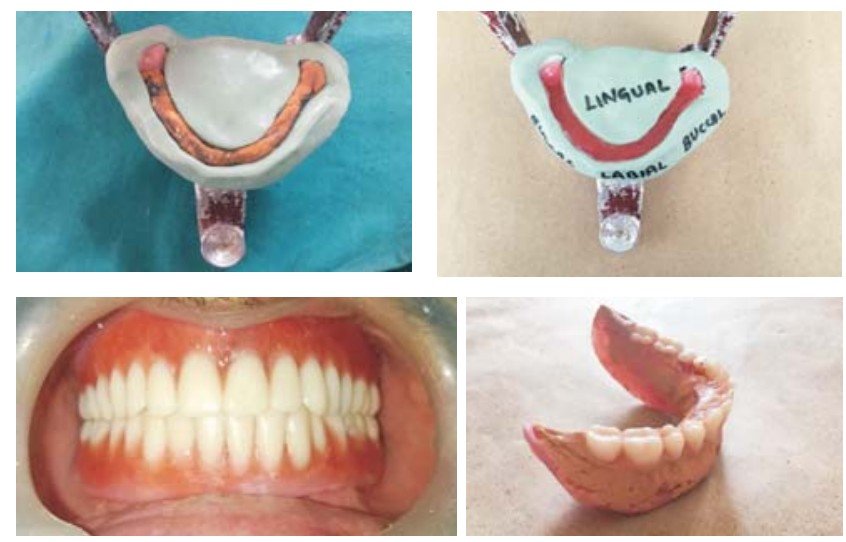

Figure 3 - Stages of incorporation of neutral zone in denture

Neutral zone limited the labial position of the mandibular anterior teeth. The mandibular posterior teeth were arranged first. They must be positioned within the neutral zone and to the proper height of the occlusal plane as established on the admixed material occlusion rim with the putty matrices in position. However position of maxillary anterior teeth could be modified based upon the esthetic and phonetic requirements of the patient. The upper posterior teeth were then positioned. Care must be taken that the occlusal surfaces of the upper posterior teeth fit perfectly against the occlusal surfaces of the lower posterior teeth (Fig.3).

Wax apical to denture teeth on the facial and lingual aspect of the mandibular trial denture was removed carefully. Then, zinc oxide eugenol impression paste was applied onto the facial aspect of mandibular trial denture and was placed into the patient's mouth. The patient was instructed to pucker the lips forward, smile broadly. The patient was asked to perform these movements repeatedly several times. The trial denture was removed and impression was evaluated. Similarly external impression of the lingual aspect of mandibular denture was made with zinc oxide eugenol impression paste as the patient was asked to do the swallowing movements. The trial denture was then removed and denture flange dimensions and extensions were examined. The excess material was trimmed off and all material covering the denture teeth was eliminated (Fig.3).

1. Lucitone(Meliodent Heat Cure,) was used as the material of choice for denture fabrication.

2. Flasking, processing, finishing and polishing of denture was then done using conventional $\operatorname{method}^{7}$ (Fig.4).

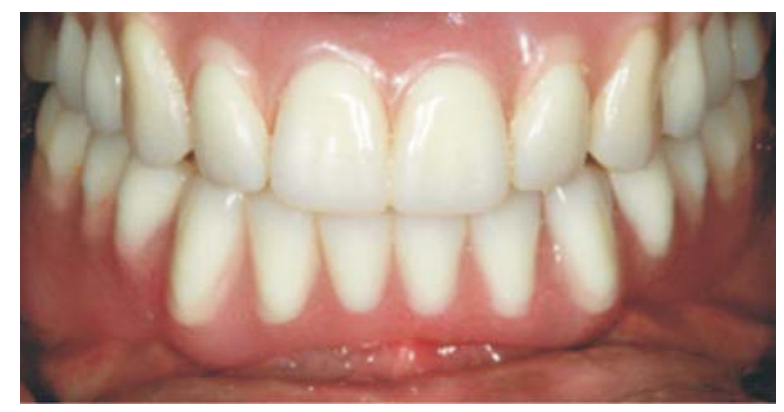

Figure 4 - Intraoral view of complete denture fabricated with neutral zone technique

\section{DISCUSSION}

The success of every complete denture relies on the fulfilment of the three basic properties of retention, stability, and support. Mandibular dentures usually present more difficulties in achieving these three properties, basically because of the larger number of anatomic limitations that requires added attention. According to Victor E. Beresin, the influence of tooth position and flange contour on denture stability is equal to or greater than that of any other factor. Teeth should be placed as dictated by the musculature, and this will vary for different patients. ${ }^{9}$ Neutral zone is an important determinant of occlusion. Failures in complete and partial dentures are often related to non-compliance with the neutral zone factors. Any part of artificial dentition out of harmony with neutral zone will result in instability, interference with function or some degree of discomfort to the patient. ${ }^{10} \mathrm{~A}$ denture shaped by neutral 
zone technique will ensure that the muscular forces are working more effectively in harmony and gives advantage of stabilizing potential oral and perioral musculature. ${ }^{11}$ Thus neutral zone plays key role in rehabilitation of edentulous patients. Using the neutral zone concept in fabricating the complete denture makes the prosthesis physiologically and psychologically more acceptable to the patient. The dentures will have other advantages: a) improved retention b) posterior will be correctly positioned allowing sufficient tongue space c) reduced food trapping adjacent to the molar teeth d) good aesthetics due to facial support. ${ }^{12}$ Various materials like tissue conditioners, Impression compound,Waxes, Impression plaster, polyethershave been advocated to record neutral zone which has their own advantages as well as disadvantages. Since tissue conditioner does not have body, one finds it difficult to use even after supporting it with wire loops.' Impression plaster is messy and cumbersome to use and fractured fragments of plaster can be swallowed by patient while performing functional movements. Uniformly reheating the modeling plastic occlusal rim is critical for its success. If this step is not performed exactly, an incorrect occlusal vertical dimension may result. ${ }^{11}$ Main disadvantage of using waxes is that they cannot record fine details, get easily distorted and also require uniform heating during their use. ${ }^{4}$ Use of polyether impression material when compared to other recommended materials for recording neutral zone is a material of choice since it has sufficient body, good flow, easy to use, less time consuming and no chance of fracturing or swallowing. ${ }^{11}$ However polyether is not economical and once set cannot be manipulated again. Advantages of using admix material is that its economical, has good flow in comparison to impression compound, thus better recording of fine details during impression making and no fractured fragments. Admixed technique ${ }^{13}$, as recommended by McCord and Tyson for atrophic mandibular ridges used, has the advantage of recording the functional position of the muscles in a single step; also it requires less chair side time. The advantages of using irreversible hydrocolloid (alginate) as wash impression with admix material during final impression of resorbed ridges is that its elastic and records good details. ${ }^{8}$ Its not correctable but can be easily remade. During neutral zone recording, patient was asked to make exaggerated "OOO" and "EEE" sounds. ${ }^{5}$ These movements flex the labial and buccal vestibular structures and mold the flanges in these regions. Swallowing molds the posterolingual flange in relation to the palatoglossus and mylohyoid musculature. Whereas, sucking flexes the labial, buccal and lingual vestibular structures and mold the flanges in these regions. Lucitone was the material of choice for denture fabrication as it is aesthetically more pleasing and is high reinforced fiber resin which provides better resistance to fracture.

\section{CONCLUSION}

This case report presents a modified approach in recording neutral zone using admix material and admixed technique. The technique described has the following advantages-the materials used for recording the neutral zone are those that are routinely used, economical and achieves the desired treatment goals. The incorporation of this simple step in fabrication of complete denture can ensure a prosthesis that is stable and retentive thereby increasing the patient comfort and satisfaction.

\section{REFERENCES}

1. Academy of Prosthodontics. The glossary of prosthodontics terms. J Prosthet Dent 2005; 94:10-92.

2. Kursoglu P, Ari N, Calikkocaoglu S. Using tissue conditioner material in neutral zone technique. N Y State Dent J 2007; 73(1):40-42.

3. Beresin VE, Schiesser FJ. The neutral zone in complete dentures. C.V.Mosby Company, Saint Louis, 1973 pp 1-150.

4. Lott F, Levin B. Flange technique: an anatomic and physiologic approach to increased retention, function, comfort and appearance of dentures. J Prosthet Dent 1966; 16:394-413.

5. Johnson A, Northeast SE. The unstable lower full denture- a practical and simple solution. Restor Dent 1989; 5:82-90.

6. Gurav SV, Khanna TS, Ghogre P. Rehabilitation of marginal 
mandibulectomy patient using neutral zone and altered cast technique. Int J Dent Health Sci 2015; 2(3):702-709.

7. Morrow RM, Rudd KD, Rhoads JE. Dental laboratory procedures-complete dentures, vol 1, 2nd edition. Mosby, St. Louis, pp 276-318.

8. Yadav B, Jayna M, Yadav H, Suri S. Comparison of Different Final Impression Techniques for Management of Resorbed Mandibular Ridge: A Case Report. Case Reports in Dentistry 2014, Article ID 253731.

9. Beresin VE, SchiesserFJ.The neutral zone in complete dentures. J Prosthet Dent 2006; 95:93-101.
10. Gupta G, Dhaded S, Goyal V, Kadian A, Gupta A. Rehabilitation of severely atrophied mandibular ridge with neutral zone technique A Case Report. IJARESM 2014; $8(2): 2455-6211$.

11. Agarwal S, Gangadhar P, Ahmad N, Bhardwaj A. A simplified approach for recording neutral zone. J IndProsthodontSoc 2010; 10(2):102-104.

12. Gahan MJ, Walmsley AD. The neutral zone impression revisited. Br Dent J 2005; 198: 269-72.

13. McCord JF, Tyson KW. A conservative prosthodontic option for the treatment of edentulous patients with atrophic (flat) mandibular ridges. Br Dent J 1997(182):469-472.

Source of Support: Nil, Conflict of Interest: None Declared 